\title{
Self-Organization of ZnO Nanoparticles on UV-Curable Acrylate Nanocomposites
}

\author{
J. A. Burunkova, I. Yu. Denisyuk, and S. A. Semina \\ Saint Petersburg State University of Information Technologies, Mechanics and Optics, Kronverskiy Avenue, 49, \\ Saint Petersburg 197101, Russia
}

Correspondence should be addressed to I. Yu. Denisyuk, denisiuk@mail.ifmo.ru

Received 14 February 2011; Revised 30 March 2011; Accepted 11 April 2011

Academic Editor: Hongmei Luo

Copyright $\odot 2011 \mathrm{~J}$. A. Burunkova et al. This is an open access article distributed under the Creative Commons Attribution License, which permits unrestricted use, distribution, and reproduction in any medium, provided the original work is properly cited.

\begin{abstract}
Our work focused on synthesis and investigation of nanoparticles role in structuring of homogenous nanocomposite based on $\mathrm{ZnO}$ nanoparticles in UV-curable monomers mixture. Due to strong interaction between nanoparticles surface and polymerizable carboxylic acid, nanoparticles were distributed homogeneously, and density of nanocomposite increased also in comparison with pure polymer matrix. Light scattering, plasticity, and water sorption non-monotonically depends on the concentration of nanoparticles concentration. UV-curable active matrix polymerization on the surface of $\mathrm{ZnO}$ nanoparticles was investigated using IR spectroscopy. The set of structural modifications of polymeric nanocomposites were observed by ASM, light scattering, Brinell hardness, and water sorption.Suggestions that the nanoparticles in the polymerization process play the role of photocatalysts and provide structuring effect on the nanocomposite were discussed.
\end{abstract}

\section{Introduction}

Nanocomposites based on metal nanoparticles and their oxides into a polymer matrix are intensively developing fields of physics and chemistry of nanosized state. Structural organization of such nanosized particles is an important problem, and without solving it, it will be difficult to make nanocomposite material suitable for practical application. The need to improve the stability of nanocomposites is attracting attention to new ways to control their morphology, structural organization, and architecture [1]. Investigation of self-regulating systems in which synthesis of the polymer matrix and the growth of nanoparticles take place simultaneously can be the best solution to the problem of stabilization of nanoparticles polymers and their structural organization. In other words, the aim is to develop methods for creating nanocomposites with architecture "microcapsulated nanoparticles in a polymer shell" formed in situ. This is done by generating a polymerized matrix of cluster dispersions, thereby limiting the growth of nanoparticles. There are many ways to do this: polymerization of vinyl monomers in the intensive mechanical dispersing of metals (initiators are freshly formed metal surface), the introduction of organometallic compounds to the polymerizing system, which decompose at temperatures close to the temperature of polymerization, $\gamma$-coirradiation of the precursor and the monomer at room temperature, polymerization of metal containing monomers, and so forth [1-3].

Nanostructured $\mathrm{ZnO}$ has attracted intensive research efforts for its unique properties and versatile applications in transparent electronics, ultraviolet (UV) light emitters, photocatalysis, photoprotecting, piezoelectric devices, chemical sensors, and spin electrics $[4,5]$. Based on these remarkable physical properties and the motivation of device miniaturization, large effort has been focused on the synthesis, characterization, and device application of $\mathrm{ZnO}$ nanomaterials $[6,7]$. In order to change the surface physicochemical properties of $\mathrm{ZnO}$ nanomaterials for the special applications, the methods such as chemical adsorption or grafting and physical wrapping were widely used [8].

There are different synthesis methods for $\mathrm{ZnO}$ nanopowder preparation [9-13]. Li et al. [9] and Vaezi and Sadrnezhaad [10] performed some studies in the field of $\mathrm{ZnO}$ nanopowder preparation via precipitation routes. They used a zinc salt and varied the $\mathrm{pH}$ and some other parameters. Chu et al. [11] used zinc acetate, citric acid, and ammonia 
and succeeded in obtaining its $\mathrm{ZnO}$ nano particles. Westin et al. [12] and Pillai et al. [13] used a sol-gel method to produce $\mathrm{ZnO}$ nanoparticles and applied the prepared powder to a varistor application and observed very high electrical properties.

In spite of the diversity of researches, there are few papers on the optical grade nanomaterials in which a high concentration of nanoparticles is combined with good optical properties.

The difficulty of these task consists in that a high concentration of structuring additives is usually accompanied by significant light scattering on them or by fluctuations in their concentration.

This work is a continuation of our research in the field of nanostructuring of polymers for optical purposes [14-16] and the last work on $\mathrm{SiO}_{2}$-based nanocomposite investigation [17]. Nanocomposites were prepared by UV curing of acrylic monomers in the presence of nanoparticles embedded in a curable matrix. Morphology of the material depends on nature and concentration of nanoparticles strongly.

The aim of the work is to study the structural changes of UV-curable polymeric $\mathrm{ZnO}$ nanocomposites and the main factors that determine their properties, such as mechanical characteristics, sorption and optical properties. Our work shows that addition of nanoparticles strongly interacting with polymer matrix will not result in worsening its homogeneity in contrary to filled polymers.

It has been found that $\mathrm{ZnO}$ nanoparticles are actively involved in the process of UV curing. Introduction in the polymerizable composition of $\mathrm{ZnO}$ nanoparticles leads to the formation of transparent and less scattering nanocomposite with good processability.

\section{Experimental}

Chemicals. We used monomers 2-Carboxyethyl acrylate (2Carb, Aldrich No. 552348), Bisphenol A glycerolate (BisA, Aldrich No. 41,116-7). For structuring nanomodification we used $\mathrm{ZnO}$ nanoparticles with a size of $20 \mathrm{~nm}$ (Russian local supplier).

Polymer films (thickness from 12 to 100 microns) were obtained from the previously prepared solutions containing monomer, nanoparticles, and its UV curing. The drop of solution is trapped between two polyester films to prevent inhibitory effects of oxygen. All experiments were accomplished at room temperature in air without special inert atmosphere. UV curing was made by a mercury lamp $(100 \mathrm{~W})$ used at the mercury line at $365 \mathrm{~nm}$.

Transmission spectra of the films were measured on a spectrophotometer Perkin-Elmer 555 UV-Vis. For the IR spectra we used Fourier IR spectrometer FSM 1201 Manufacturer Company "Monitoring." Samples were prepared by pressing pellets with $\mathrm{KBr}$.

Refractive index is measured on an Abbe refractometer in accordance with the recommendations of the European standard ASTM D542.

In this paper we study sorption of water vapor by gravimetric method.
Hardness is measured by Brinell hardness method with "Bulat-T1" device.

Light scattering is measured by the photometric sphere method in accordance with the recommendations of the European standard ASTM D1003.

Investigation of the surface profile of samples was made with an atomic force microscope Ntegra used in contact mode.

\section{Results}

We find some effects at mixing of nanoparticles with monomers mixture. At $\mathrm{ZnO}$ introduction in the composition of the monomer BisA/2Carb (30/70) above a concentration of $8 \mathrm{wt} . \%$ the viscosity of solutions increases greatly. At higher concentrations ( $14 \mathrm{wt} . \%$ and higher) viscosity of the solution continues to grow and the mixture becomes solid at room temperature.

Transparent nanocomposites were obtained up to $14 \mathrm{wt} . \%$ of $\mathrm{ZnO}$ nanoparticles with formation of transparent film after UV curing. More than $14 \mathrm{wt} . \%$ of $\mathrm{ZnO}$ particles addition to the monomer mixture resulted in turbid films. The system becomes heterogeneous.

The films are transparent in the visible and UV spectral region. A significant decrease in optical transmission is observed at high concentrations of $\mathrm{ZnO}$ (more than 14 wt.\%).

The calculated (1) and experimental (2) values of the refractive index nanocompositions are shown in Figure 1(a). For the theoretical calculation of the refractive index, Maxwell-Garnett's model of effective medium has been used [18]:

$$
\frac{\varepsilon_{\text {eff }}-\varepsilon_{2}}{\varepsilon_{\text {eff }}+2 \varepsilon_{2}}=f_{1} \frac{\varepsilon_{1}-\varepsilon_{2}}{\varepsilon_{1}+2 \varepsilon_{2}},
$$

Where $\varepsilon_{1}$ is the permittivity of the medium, $\varepsilon_{2}$ is the permittivity of inclusions, $\varepsilon_{\text {eff }}$ is the permittivity of the composite medium, $f_{1}=(1 / V) \Sigma_{i} V_{i}$ is the volumetric filling factor, ( $V_{i}$ is the volume of $i$ th particle, $V$ is the volume of the composite environment).

This model is applicable when the volume filling factor: $f_{1} \leq 1 / 3$, that is, fraction of inclusions is small. The experimentally obtained values for the film samples are higher than theoretically calculated. This effect can be explained by the following: according our recent results, there is interaction between nanoparticles and polymer matrix. Model of Maxwell-Garnett is applicable in the case when nanoparticles are distributed in any matrix. As a result of interaction between nanoparticles and polymer matrix this model condition is not accomplished. [19]. Refractive index of composition with maximum $\mathrm{ZnO}$ concentration ( $14 \mathrm{wt} . \%$ ) is increased by 0.045 compared with the polymer without nanoparticles.

Figure $1((\mathrm{~b})$, curve 3$)$ shows that at increasing $\mathrm{ZnO}$ concentration, scattering of nanocomposite decreases, but near $4 \mathrm{wt} . \%$ there is a maximum of scattering. We think that near this concentration takes place a reorganization of polymer inner structure from polymeric structure with 


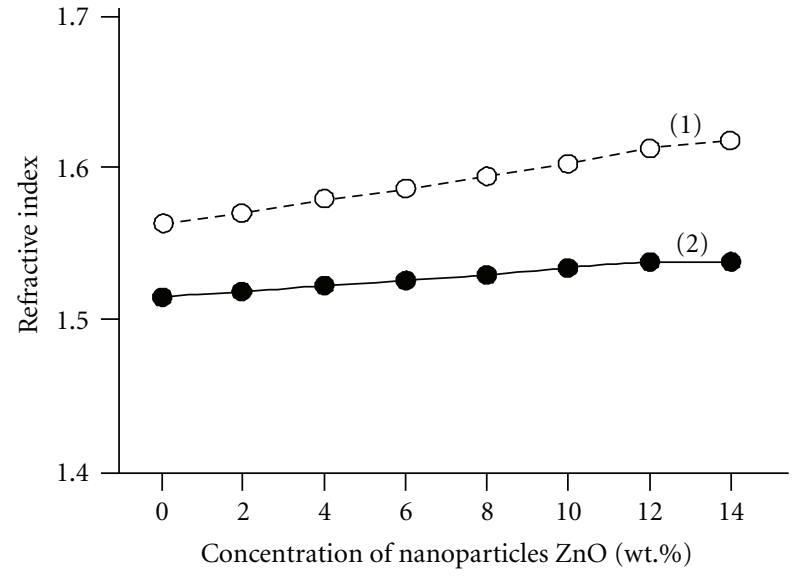

(a)

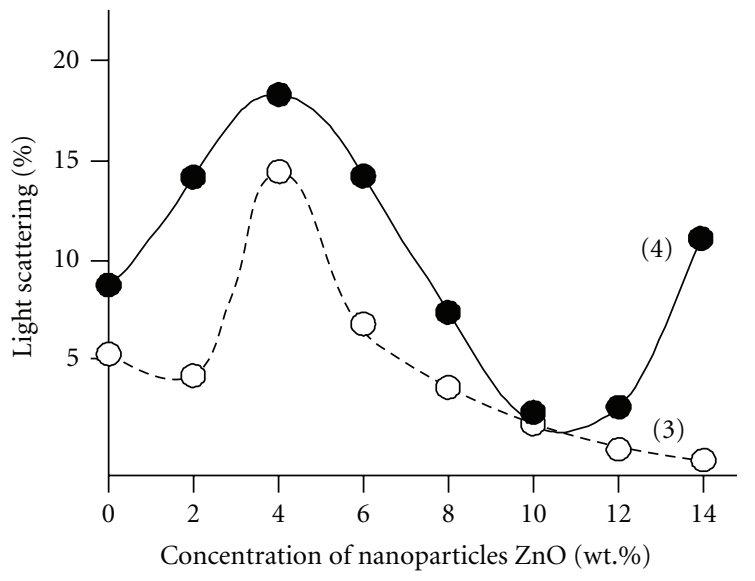

(b)

Figure 1: Refractive index (a): experimental RI (1) and calculation RI (2), light scattering (b): before (3) and after (4) water sorption of the composition BisA/2Carb (30/70) versus $\mathrm{ZnO}$ nanoparticles concentrations.

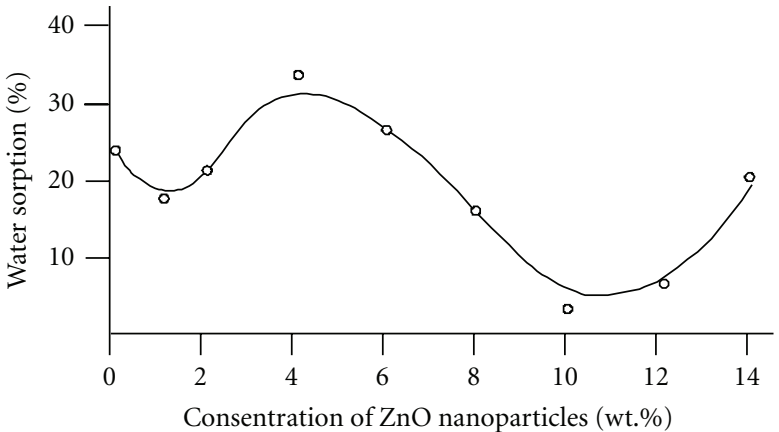

(a)

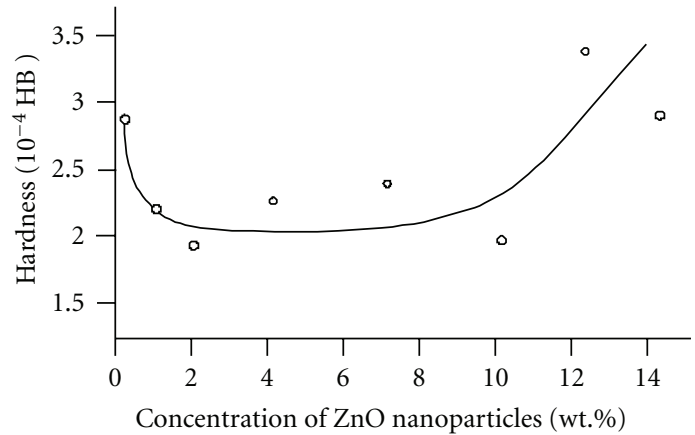

(b)

FIgURE 2: Sorption of water (a) and hardness (b) of composite BisA/2Carb (30/70) versus concentrations of $\mathrm{ZnO}$.

inclusion of nanoparticles to self-organized nanocomposite structure (see Figure 3).

When the concentration of $\mathrm{ZnO}$ is more than $14 \mathrm{wt} . \%$, light scattering is independent of the concentration of nanoparticles. $\mathrm{ZnO}$ amount is sufficient for uniform distribution in volume, and homogeneous polymer composites are formed. In this structure, the light scattering decreases compared to pure polymer, approximately twice.

The water sorption experiments were conducted to study changes in the internal volume of the polymer as a result of effect of free volume filling by $\mathrm{ZnO}$ nanoparticles (Figure 1 ((b), curve 4)).

Water sorption properties of nanocomposite have strong dependence on nanoparticles concentration (Figure 2(a)). There is a sorption maximum near $4 \%$ (reorganization of polymer inner structure). At higher nanoparticles concentration (12 wt. \% $\mathrm{ZnO}$ ) water sorption increases, perhaps as a result of disordering of the nanocomposite.

For pure polymer value, water sorption is $23 \%$. The introduction of $10 \mathrm{wt} . \%$ nanoparticles achieved reduction in water sorption by 5 times compared with the pure composition.
The hardness investigation of films is an indirect way to study the effect of $\mathrm{ZnO}$ on the structural change of nanocomposites (Figure 2(b)).

At the introduction of $\mathrm{ZnO}$ up to $10 \mathrm{wt} . \%$ the hardness of the films decreases. Further, the hardness of the film increases and reaches the one of pure polymer.

The changes of the polymer nanocomposite properties (water sorption, light scattering, hardness) confirmed that nanoparticles influence on the polymer structure by means of possible interactions of $\mathrm{ZnO}$ with active groups of the polymer [20].

The investigation of the surface relief of the nanocomposites made by atomic force microscopy is shown in Figure 3.

As can be seen from Figure 3, there are essential changes in the composition structure as compared with the original by introduction at $4 \mathrm{wt}$. $\% \mathrm{ZnO}$ nanoparticles. The formation of separate polymer regions of structured $\mathrm{ZnO}$ was observed clearly. The grain uniform structure are observed throughout the material when $\mathrm{ZnO}$ concentration achieves $10 \mathrm{wt} . \%$. Apparently, formation of this structure is due to the ability of nanoparticles to create weak bonds with the active groups 

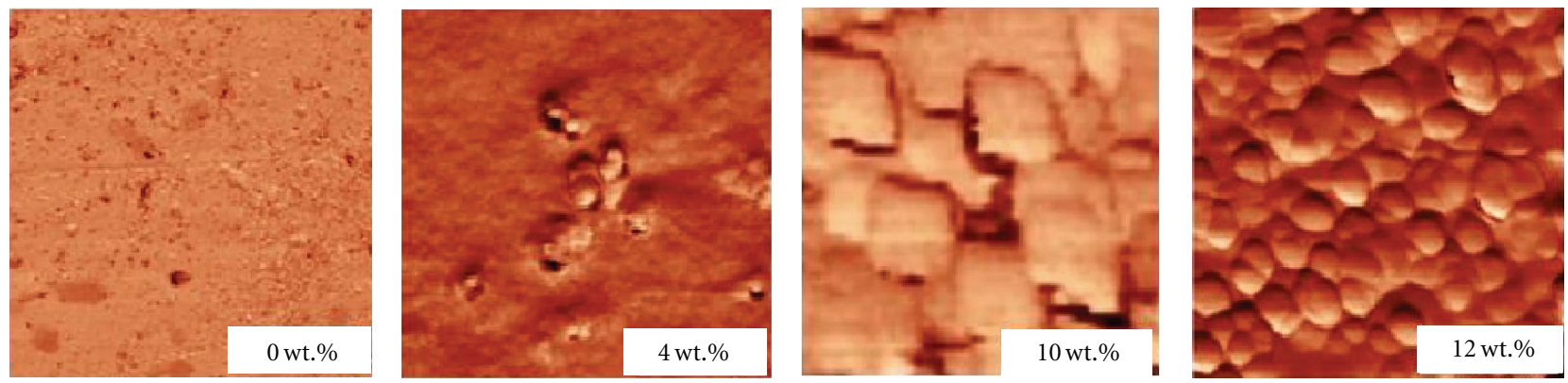

BisA/2Carb (30/70) + ZnO nanoparticles (wt.\%)

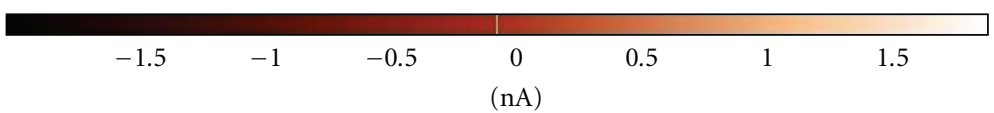

FIGURE 3: Relief of polymer films with the introduction of 0 wt.\% (a), 4 wt.\% (b), 8 wt.\% (c), 12 wt.\% (d) ZnO nanoparticles (AFM). The size of photos $5 \times 5 \mathrm{mkm}$.

of the monomer molecules and to work as centers of polymerization.

When the $\mathrm{ZnO}$ concentration is more than $10 \mathrm{wt} . \%$, free polymer phase disappears and all available polymers form spheres on nanoparticles surface.

At low concentrations of nanoparticles, modified polymer areas are not enough and composites are heterogeneous. Heterogeneous structures are reflected in their properties. With increasing $\mathrm{ZnO}$ concentration, size and number of hybrid fields are growing, thus it is conditioned by forming of a completely different structure of the material.

The formation of submicron spheres around each nanoparticle is the cause of formation of quasi-homogeneous materials, in which light scattering does not exceed the pure polymer. Indeed (Figure 3), submicron spheres formed around each nanoparticle possess almost identical diameters. This fact can be explained by identical growth rates of these spheres. The result is structure consisting of sphere particles located in the self-organized quasilattice. Eventually, the above-named effects lead to a homogeneous distribution of nanoparticles and the formation of a homogeneous environment.

Determination of possibility and mechanism of polymerization on surface of $\mathrm{ZnO}$ nanoparticles were investigated by mean of FTIR nanocomposite spectra (Figure 4). FTIR spectra of monomers mixture BisA/2Car (30/70) may be seen as peaks at 1737,1410 and $1188,1050 \mathrm{sm}^{-1}$. The first peak corresponds to valency vibration of carboxylic group, next three-to combination of flat deformation vibration of hydroxide group and valency vibration of C-O links in carboxylic acids [21].

At preparation of nanocomposite FTIR spectra change essentially, peak $1720 \mathrm{sm}^{-1}$ (C-O group) keep and peak $1410-1450 \mathrm{sm}^{-1}$ (valency vibration of carboxylate anion) will intensify. It is important to notice that the peak at $500 \mathrm{sm}^{-1}$ corresponds to $\mathrm{Zn}-\mathrm{O}$ vibration. A peaks appears between $1620-1550 \mathrm{sm}^{-1}$. This area is typical of asymme-tric valency vibration of carboxylic anion. According to informa-

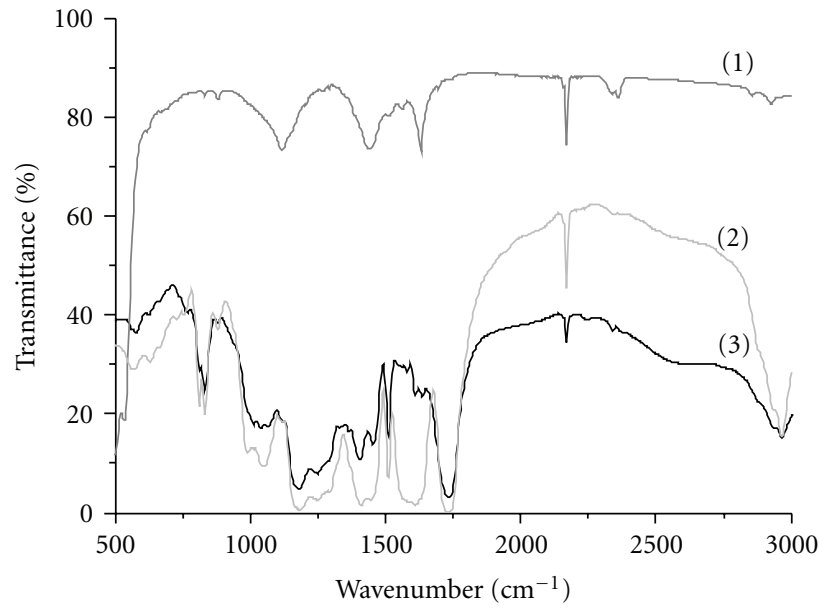

FIGURE 4: FTIR spectra: nanoparticles $\mathrm{ZnO}$ (1), composition 2Carb/BisA (70/30) + $12 \mathrm{wt} . \% \mathrm{ZnO}$ (2), and pure 2Carb/BisA $(70 / 30)(3)$.

tion of works [22-25] interaction of inorganic nanoparticles $\mathrm{ZnO}$ and carbonyl group results in change in IR spectra, as metal atoms can be connected to carbonyl oxygen by electron pair transfer. The increase of peak intensity $\mathrm{O}-\mathrm{H}$ at $1550 \mathrm{sm}^{-1}$ can be a result of formation of a complex between the polymer and nanoparticle.

Next, in nanocomposite peaks appearing at 1640$1650 \mathrm{sm}^{-1}$, they are typical for $\mathrm{C}=\mathrm{C}$ valency vibration in $\mathrm{CH}_{2}=\mathrm{CHR}$, and peak $990 \mathrm{sm}^{-1}$ is typical for C-H deformation vibration in $\mathrm{CH}_{2}=\mathrm{CHR}$. $\mathrm{CH}_{2}=\mathrm{CHR}$ groups can photopolymerize on $\mathrm{ZnO}$ surface, acting as photoinitiator. Principal scheme of described process is shown in Figure 5.

\section{Discussion on Obtained Results}

Our results show that $\mathrm{ZnO}$ nanoparticles can be involved in UV polymerization process with formation of bonds 


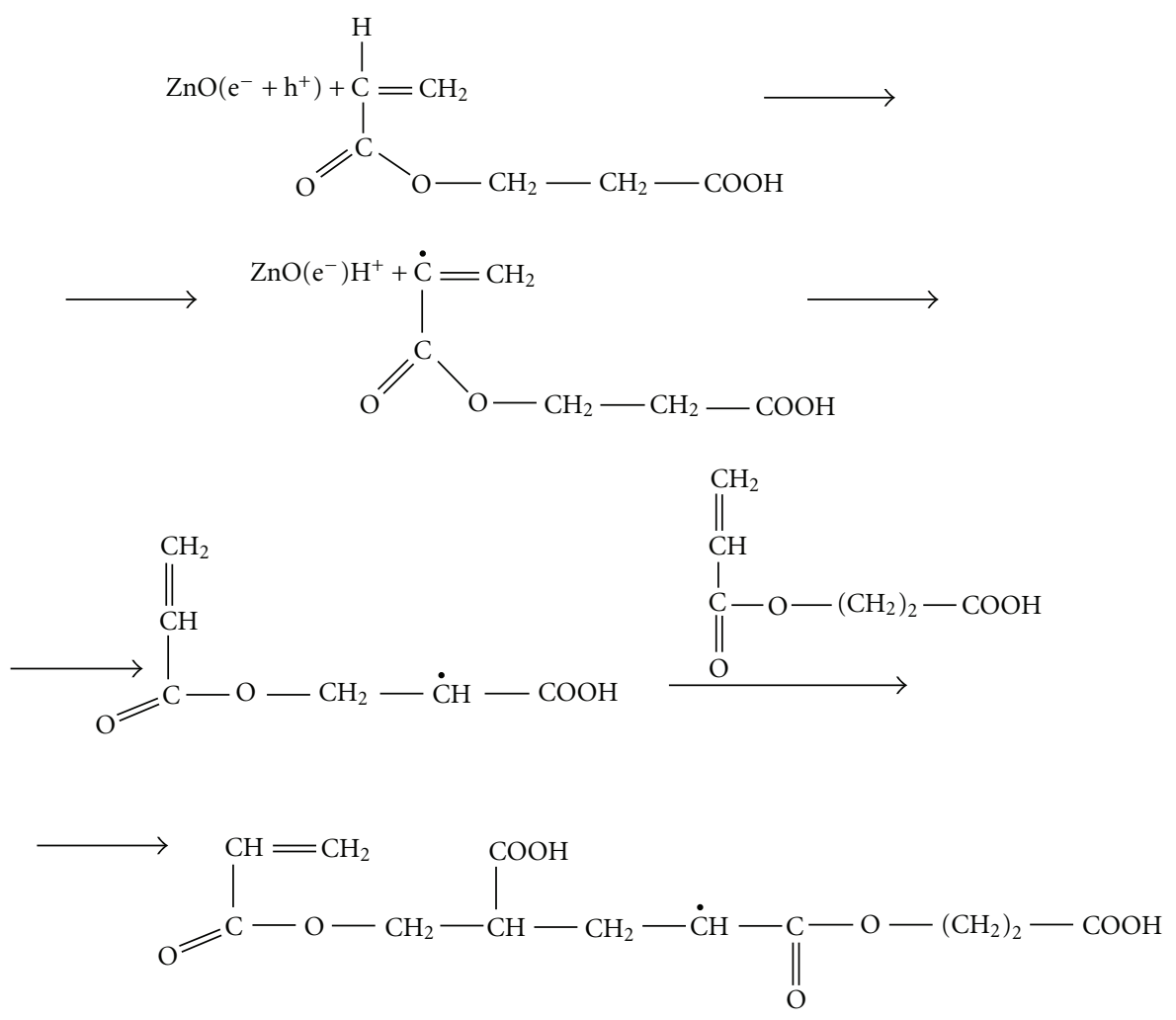

FIGURE 5: Scheme of photocatalysis process.

between nanoparticle surfaces and polymers. Thus, the $\mathrm{ZnO}$ nanoparticles act as centers of formation of a new polymer phase-the nanocomposite, other than with the pure polymer properties.

According to FTIR spectra of $\mathrm{ZnO}$ nanocomposites we assume that nanoparticles act as photocatalysts in polymerization and became as a centers of polymerization as a result of interaction with carboxylic group of one of the composition monomers. Our hypotheses are proved by formation of micron size spheres in nanocomposite around each nanoparticle (Figure 3). Nanosized semiconductor clusters have the potential of photooxidation and photocatalysis through the combined effects of photoelectrons production at UV light absorption and high surface area in which electron-transferinduced polymerization takes place. According to results of the work in [26], during the photocatalytic process, the illumination of a semiconductor photocatalyst with ultraviolet (UV) radiation activates the catalyst, establishing a redox environment in the aqueous solution. Semiconductors act as sensitizers for light-induced redox processes due to their electronic structure, which is characterized by a filled valence band and an empty conduction band. So, light absorption by semiconductor nanoparticles will result in photocatalytic process in its surface including photopolymerization processes. The work in [27] shows possibility of this process of methyl methacrylate photopolymerization initiation by $\mathrm{TiO}_{2}$ nanoparticles. In our experiments we obtain similar process that is proved by FTIR and atomic force microscopy results (formation of equal spheres around each nanoparticle as a center of polymerization and chemical bonding between nanoparticles surface and polymer).

Formation of spheres around each nanoparticles as a result of photopolymerization on its surface results in transformation of polymer structure: at augmentation of nanoparticles concentration all proprieties of composition have sudden extremums at the same values of nanoparticles concentration. Characteristic points are 4 and $10 \mathrm{wt} \%$. According AFM photos between 2 and $4 \mathrm{wt} \%$ of nanoparticles, new phase of polymer generated on nanoparticles surface will occupy mostly part of nanocomposite volume. It results in structuring of material, light scattering, and water sorption will decrease. At nanoparticles concentration more than $10 \mathrm{wt} \%$, all volume of polymers will be involved in formation of new phase; perhaps next increasing of nanoparticles concentration will result in competition between nanoparticles as the center of polymerization resulted in missequencing of material. AFM photo shows that structures consisting of micro spheres occupy all volume, and light scattering and water sorption increase. Above-listed effects are proved by missequencing of nanocomposite.

Certainly these suggestions are our hypothesis only. Future investigation of nanocomposite with active nanoparticles is needed to understand the causes of these strange effects of composite reorganization. 


\section{Conclusion}

The sorption of water vapor, Brinell hardness, optical transmission, refractive index, and light scattering of film polymer $\mathrm{ZnO}$ nanocomposites were studied.

Composites are transparent in the visible spectrum at high concentrations of $\mathrm{ZnO}$ nanoparticles (14 wt.\%). With the introduction of $14 \mathrm{wt} . \% \mathrm{ZnO}$ the refractive index composite increases to 0.045 . With the introduction of $10 \mathrm{wt} . \% \mathrm{ZnO}$ the sorption decreases five times. Hardness, until a maximum concentration of nanoparticles $12 \mathrm{wt} . \%$ $\mathrm{ZnO}$, does not exceed the hardness of the pure polymer, while light scattering is not increased.

Nonmonotonic changes in the properties, the AFM data, and the IR spectra demonstrate the ability of nanoparticles to act as centers of polymerization and to form a granular structure in the nanocomposite.

\section{Acknowledgment}

The work was made under support of Russian Ministry of Education Grant GK P995 under Federal Program "Teaching and scientific personnel of innovative Russia,” 2009-2013.

\section{References}

[1] A. S. Rosenberg, G. I. Dzhardimalieva, and A. D. Pomogailo, "Polymer composites of nano-sized particles isolated in matrix," Polymers for Advanced Technologies, vol. 9, no. 8, pp. 527-535, 1998.

[2] A. D. Pomogailo and V. S. Savost'yanov, Synthesis and Polymerization of Metal-Containing Monomers, CRC Press, Boca Raton, Fla, USA, 1994.

[3] A. S. Pomogailo, A. S. Rozenberg, G. I. Dzhardimalieva, and M. Leonowicz, "Polymer nanocompositeson the baseof metal carboxylates," Advances in Materials Science, vol. 1, no. 1, pp. 19-27, 2001.

[4] M. J. Height, S. E. Pratsinis, O. Mekasuwandumrong, and P. Praserthdam, "Ag-ZnO catalysts for UV-photodegradation of methylene blue," Applied Catalysis B, vol. 63, no. 3-4, pp. 305312, 2006.

[5] L. Guedri-Knani, J. L. Gardette, M. Jacquet, and A. Rivaton, "Photoprotection of poly(ethylene-naphthalate) by zinc oxide coating," Surface and Coatings Technology, vol. 180-181, pp. 71-75, 2004.

[6] Z. Y. Fan and J. G. Lu, "Zinc oxide nanostructures: synthesis and properties," Journal of Nanoscience and Nanotechnology, vol. 5, no. 10, pp. 1561-1573, 2005.

[7] X. Fang, Y. Bando, U. K. Gautam et al., "ZnO and ZnS nanostructures: ultraviolet-light emitters, lasers, and sensors," Critical Reviews in Solid State and Materials Sciences, vol. 34, no. 3-4, pp. 190-223, 2009.

[8] P. Liu and T. Wang, "Poly(hydroethyl acrylate) grafted from $\mathrm{ZnO}$ nanoparticles via surface-initiated atom transfer radical polymerization," Current Applied Physics, vol. 8, no. 1, pp. 6670, 2008.

[9] Y. Li, G. Li, and Q. Yin, "Preparation of $\mathrm{ZnO}$ varistors by solution nano-coating technique," Materials Science and Engineering B, vol. 130, no. 1-3, pp. 264-268, 2006.
[10] M. R. Vaezi and S. K. Sadrnezhaad, "Nanopowder synthesis of zinc oxide via solochemical processing," Materials \& Design, vol. 28, no. 2, pp. 515-519, 2007.

[11] S.-Y. Chu, T.-M. Yan, and S.-L Chen, "Analysis of ZnO varistors prepared by the sol-gel method," Ceramics International, vol. 26, no. 7, pp. 733-737, 2000.

[12] G. Westin, A. Ekstrand, M. Nygren, R. Österlund, and P. Merkelbach, "Preparation of $\mathrm{ZnO}$-based varistors by the solgel technique," Journal of Materials Chemistry, vol. 4, no. 4, pp. 615-621, 1994.

[13] S. C. Pillai, J. M. Kelly, D. E. McCormack, and R. Ramesh, "Self-assembled arrays of $\mathrm{ZnO}$ nanoparticles and their application as varistor materials," Journal of Materials Chemistry, vol. 14, no. 10, pp. 1572-1578, 2004.

[14] M. I. Fokina, I. Yu. Denisyuk, Y. É. Burunkova, and L. N. Kaporskiü, "Formation of microstructures based on UVhardenable acrylates," Journal of Optical Technology, vol. 75, no. 10, pp. 664-669, 2008.

[15] I. Yu. Denisyuk, T. R. Williams, and J. E. Burunkova, "Hybrid optical material with nanoparticles at high concentrations in UV-curable polymers-technology and properties," Molecular Crystals and Liquid Crystals, vol. 497, pp. 142-153, 2008.

[16] T. R. Williams, I. Yu. Denisyuk, and J. E. Burunkova, "Filled polymers with high nanoparticles concentration-synthesis, optical and rheological proprieties," Journal of Applied Polymer Science, vol. 116, no. 4, pp. 1857-1866, 2010.

[17] J. A. Burunkova, I.Yu. Denisyuk, N. N. Arefeva, and S. A. Semena, "Influence of $\mathrm{SiO}_{2}$ nanoaddition on the selforganization via UV-polymerization of acrylate nanocomposites," Molecular Crystals and Liquid Crystals, vol. 536, no. 1, pp. 10-16, 2011.

[18] A. P. Vinogradov, Electrodynamics of Composite Materials, Aditorial URSS, Moscow, Russia, 2001.

[19] J. E. Spanier and I. P. Herman, "Use of hybrid phenomenological and statistical effective-medium theories of dielectric functions to model the infrared reflectance of porous $\mathrm{SiC}$ films," Physical Review B, vol. 61, no. 15, pp. 10437-10450, 2000 .

[20] S. Jiguet, A. Bertsch, M. Judelewiczb, H. Hofmannc, and P. Renaud, "SU-8 nanocomposite photoresist with low stress properties for microfabrication applications," Microelectronic Engineering, vol. 83, no. 10, pp. 1966-1970, 2006.

[21] R. M. Silverstein, G. C. Bassler, and T. C. Morrill, Spectrometric Identification of Organic Compounds, John Wiley \& Sons, New York, NY, USA, 4th edition, 1981.

[22] X. Lu, Y. Zhao, and C. Wang, "Fabrication of PbS nanoparticles in polymer-fiber matrices by electrospinning," Advanced Materials, vol. 17, no. 20, pp. 2485-2488, 2005.

[23] X. Lu, Y. Zhao, C. Wang, and Y. Wei, "Fabrication of CdS nanorods in PVP fiber matrices by electrospinning," Macromolecular Rapid Communications, vol. 26, no. 16, pp. 1325-1329, 2005.

[24] J. Bai, Y. Li, C. Zhang, X. Liang, and Q. Yang, "Preparing AgBr nanoparticles in poly(vinyl pyrrolidone) (PVP) nanofibers," Colloids and Surfaces A, vol. 329, no. 3, pp. 165-168, 2008.

[25] A. H. Kupcov and G. N. Jijin, Raman Scattering and Fourier -IR Polymers Spectra, Fismatlit, Moscow, Russia, 2001.

[26] D. Beydoun, R. Amal, G. Low, and S. McEvoy, "Role of nanoparticles in photocatalysis," Journal of Nanoparticle Research, vol. 1, no. 4, pp. 439-458, 1999.

[27] C. Dong and X. Ni, "The photopolymerization and characterization of methyl methacrylate initiated by nanosized titanium dioxide," Journal of Macromolecular Science, Part A, vol. 41, no. 5, pp. 547-563, 2004. 

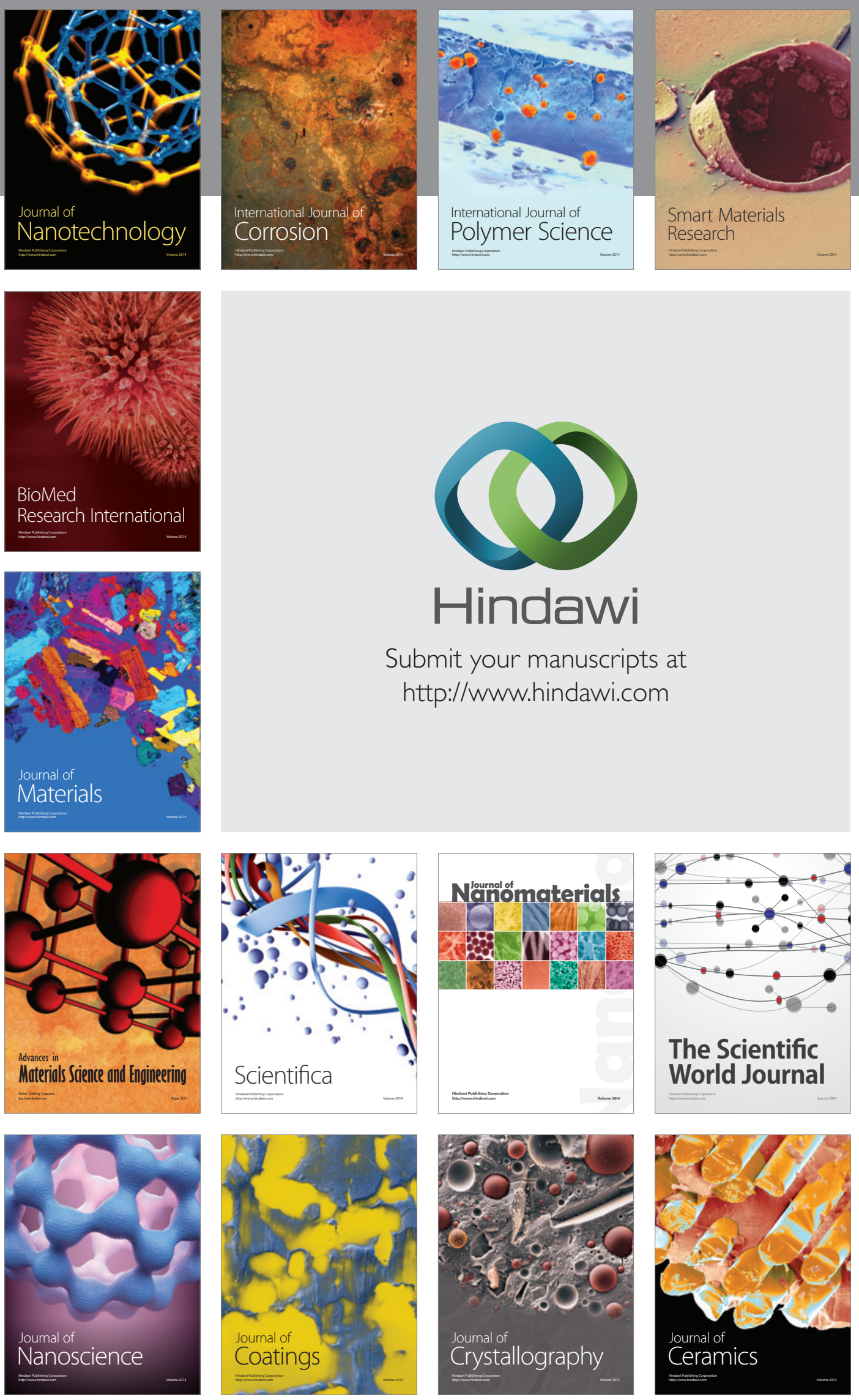

The Scientific World Journal

Submit your manuscripts at

http://www.hindawi.com

\section{World Journal}

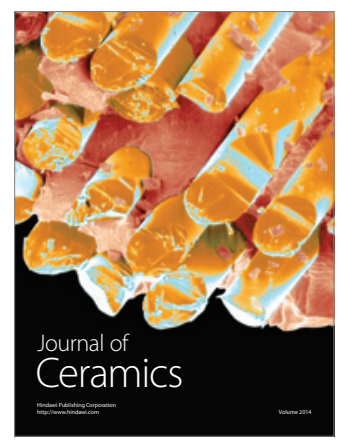

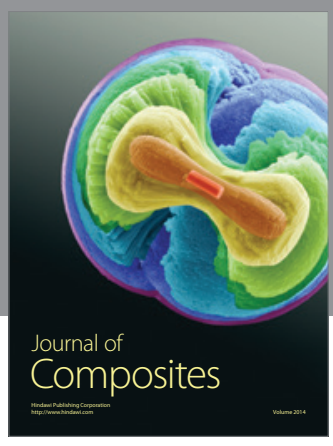
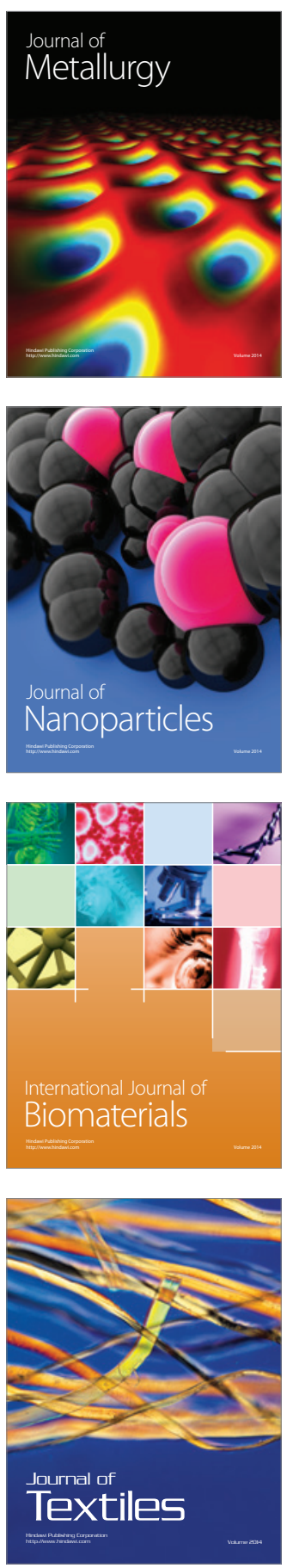